\title{
Left ventricle remodeling predicts the recurrence of ventricular tachyarrhythmias in implantable cardioverter defibrillator recipients for secondary prevention
}

Wei-Chieh Lee ${ }^{1}$, Huang-Chung Chen ${ }^{1}$, Yung-Lung Chen ${ }^{1}$, Tzu-Hsien Tsai ${ }^{1}$, Kuo-Li Pan², Yu-Sheng Lin² and Mien-Cheng Chen ${ }^{1 *}$

\begin{abstract}
Background: Implantable cardioverter defibrillator (ICD) is an effective treatment for secondary prevention of ventricular tachycardia/ventricular fibrillation (VTNF). Left ventricular (LV) remodeling may develop before ICD implant and over time. However, it remains unclear how LV remodeling affects subsequent risk for recurrence VTNF in ICD recipients under optimal medical therapy.

Methods: From May of 2004 to June of 2015, 144 patients received ICD implantation for secondary prevention were enrolled in this study. All information interrogated from ICD devices during follow-up or ICD therapy history (antitachycardia pacing and shock therapy) were reviewed and validated the occurrences of VTNF.

Results: At a mean follow-up of $1110.5 \pm 860.6$ days, 53 patients (36.8\%) had recurrence of VTNF episodes and 91 patients had no recurrence of VTNF episode after ICD implant. Left ventricular end-diastolic volume (LVEDV) $>163.5 \mathrm{~mL}$ had significant predictive value for VTNF recurrence (area under the curve: $0.602, p=0.041$ ). Moreover, the percentage of patients with LVEDV $>163.5 \mathrm{~mL}$ was significantly higher in patients with recurrent VTNF than patients without recurrent VTNF (62.3 vs 40.0\%, $p=0.010)$. Left ventricular ejection fraction $\leq 30 \%(p=0.031)$, LVEDV $>163.5 \mathrm{~mL}(p=0.012)$ and QRS width $>125 \mathrm{msec}(p=0.049)$ were significant predictors for VTNF recurrence by univariate Cox regression analysis. However, only LVEDV > $163.5 \mathrm{~mL}$ (hazard ratio: 2.549, 95\% confidence interval: 1.249 5.201, $p=0.010$ ) and QRS width $>125$ msec (hazard ratio: 2.173, 95\% confidence interval: $1.030 \sim 4.586, p=0.042$ ) were independent predictors for recurrence of VTNF after multivariable adjustment.
\end{abstract}

Conclusion: LV remodeling and QRS width > 125 msec were independent predictors for VTNF recurrence in secondary prevention ICD recipients under optimal medical therapy, independent of LV ejection fraction.

Keywords: Implantable cardioverter defibrillator, Ventricular remodeling, Ventricular tachycardia

\section{Background}

Implantable cardioverter defibrillator (ICD) has been confirmed to be an effective treatment for selected patients who are survivors of sustained ventricular tachycardia/ventricular fibrillation (VT/VF) unrelated to transient correctable causes or are at risk of sudden

\footnotetext{
* Correspondence: chenmien@ms76.hinet.net

'Division of Cardiology, Department of Internal Medicine, Kaohsiung Chang Gung Memorial Hospital, Chang Gung University College of Medicine, 123 Ta Pei Road, Niao Sung District, Kaohsiung City 83301, Taiwan

Full list of author information is available at the end of the article
}

cardiac death [1]. Several studies have consistently confirmed the preponderance of ICD therapy over antiarrhythmic therapy in terms of primary or secondary prevention of sudden cardiac death and mortality in patients with heart failure and reduced left ventricular (LV) function [2-7]. However, ICD therapy is an expensive therapy and is reimbursed only for secondary prevention in many developing countries. Additionally, some ICD recipients for primary or secondary prevention may never experience recurrence of VT/VF and shock therapy during follow-up under optimal medical therapy, 
including $ß$-blockers and renin-angiotensin-aldosterone antagonists, and modification of risk factors. Therefore, clinical predictors for recurrent VT/VF as the guidance for ICD replacement are requisite for limited healthcare resources in many developing countries. The majority of studies of clinical predictors for recurrent VT/VF enrolled both primary and secondary prevention ICD recipients. Klein et al. reported that LV ejection fraction < $40 \%$, permanent atrial fibrillation and QRS duration > $150 \mathrm{msec}$ are independent predictors for VT/VF recurrence in ICD recipients for primary and secondary prevention [8]. However, there are limited reports regarding clinical predictors for recurrent VT/VF in patients received ICD only for secondary prevention. Interestingly, Freedberg et al. reported no predictor for subsequent ICD therapy in patients receiving ICD for symptomatic VT or cardiac arrest [9].

Left ventricular remodeling associated with underling heart disease may develop before ICD implant and over time after implant. Moreover, $ß$-blockers and reninangiotensin-aldosterone antagonists have been shown to inhibit or reverse LV remodeling rather than changes in LV ejection fraction [10]. However, it remains unclear how LV remodeling affects subsequent risk for recurrence VT/ VF in ICD recipients for secondary prevention. Accordingly, we conducted this study to investigate the role of LV remodeling as a predictor for the recurrence of VT/VF in ICD recipients for secondary prevention under optimal medical therapy.

\section{Methods}

\section{Patient population}

From May of 2004 to June of 2015, 144 patients received ICD implantation for secondary prevention were enrolled in this study. All information interrogated from ICD devices during follow-up or ICD therapy history (anti-tachycardia pacing and shock therapy) were reviewed and validated the occurrences of VT/VF and ICD therapy. Ninety-one patients who did not have any VT/VF episode or ICD therapy during follow-up after ICD implant were defined as the "no recurrent VT/VF group", whereas 53 patients who had experienced VT/VF episodes and appropriate ICD therapies during follow-up after implant were defined as the "recurrent VT/VF group".

\section{Definitions}

Left ventricular diastolic dysfunction was categorized into 3 stages: stage I (impaired relaxation), stage II (pseudonormalization) and stage III (restrictive filling pattern) according to the echocardiographic patterns of mitral inflow velocity, mitral Doppler tissue imaging of mitral annular motion and pulmonary venous flow. The LV end-diastolic volume (LVEDV) and LV end-systolic volume (LVESV) were quantified by M-mode echocardiography and were corrected by two-dimensional guided biplanar Simpson's method of discs for measurement $[11,12]$.

Atrial fibrillation was defined as paroxysmal if atrial fibrillation episode lasting for 7 days or less, persistent if continuous atrial fibrillation episode lasting for more than 7 days, and longstanding persistent if continuous atrial fibrillation episode lasting for more than 1 year.

\section{Study endpoints}

The primary study endpoints were the recurrence of VT/VF and ICD therapy. The secondary endpoints were cardiovascular death (death related to heart failure and cardiac arrhythmia) and all-cause mortality (including sepsis, hepatic failure and brain hemorrhage).

\section{Statistical analysis}

Data are presented as mean \pm SD or percentage. The clinical characteristics (general demographics, underling heart diseases, comorbidities, functional class of heart failure, QRS duration, medications, LVEDV, LVESV, LV function, VT/VF detection zone and VT ablation) between the study groups were compared using $t$-test for continuous variables or chi-square test for categorical variables. Receiver operating characteristic curve analysis was used to calculate the area under the curve for the optimal volume of LVEDV, LVESV and QRS width in predicting the recurrence of VT/ VF. Univariate and multivariate Cox regression analyses were performed to identify the significant predictors for the recurrence of VT/VF after implant. Each independent variable was based on previous studies and conventional risk factors, and was expressed as a hazard ratio with $95 \%$ confidence interval. The Kaplan-Meier method and log-rank test were used to compare the event-free survival of the recurrence of VT/VF during follow-up. Statistical analysis was performed using statistical software (SPSS for Windows, version 22). A two-sided $p$ value of $<0.05$ was considered statistically significant.

\section{Results \\ Baseline characteristics of study patients}

At a mean follow-up of $1110.5 \pm 860.6$ days, 53 patients (36.8\%) had recurrence of VT/VF episodes and 91 patients had no recurrence of any VT/VF episode after ICD implant (Fig. 1). Table 1 lists the clinical characteristics of the study group. In this study, $71.5 \%$ of patients received renin-angiotensin-aldosterone antagonists and $60.4 \%$ of patients received $\beta$-blocker therapy. The average age of patients in recurrent VT/VF group was $60 \pm 13$ years, and $77.4 \%$ of the patients were male. The average age of patients in no recurrent VT/VF group was $63 \pm 14$ years, and $80.2 \%$ of the patients were male. Patients with coronary artery disease in recurrent VT/VF group had higher prevalence of revascularization with coronary artery bypass graft surgery than patients with coronary artery 


\section{VT/VF recurrence}

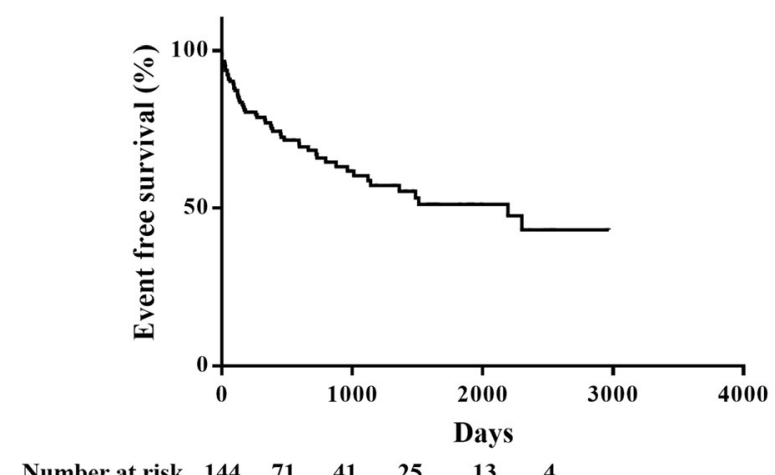

$\begin{array}{lllllll}\text { Number at risk } & 144 & 71 & 41 & 25 & 13 & 4\end{array}$

Fig. 1 Kaplan-Meier plot proportion of patients free of recurrent ventricular tachycardia/ventricular fibrillation (VTNF) events after implant in the study cohort

disease in no recurrent VT/VF group. There was no significant difference in age, gender, body mass index, underling heart diseases, prevalence of comorbidities and atrial fibrillation, frequency of medications related to comorbidities and anti-arrhythmics, the average dosage of ß-blocker and anti-arrhythmics, QRS duration, the percentages of left bundle branch block and ventriculardependent pacing, stage of LV diastolic dysfunction and heart failure status between the two groups. There was no significant difference in the prevalence of unstable VT ( $p$ $=0.590$ ) and VT ablation plus medications (anti-arrhythmics and $\beta$-blocker) ( $p=0.358)$ between the two groups. Seventy nine patients received single chamber ICD and 65 patients received dual chamber ICD. There was no significant difference in the ICD type and the percentage of cardiac resynchronization therapy defibrillator recipients between the two groups. The lowest VT- and VFdetection rates were similar between two groups. Fifteen patients (10.4\%) received catheter ablation and modification of arrhythmic substrates, including 6 patients (6.6\%) in no recurrent VT/VF group, and 9 patients (17.0\%) in recurrent VT/VF group $(p=0.087)$ (Table 1$)$.

\section{Determinants of recurrence of VT/VF during follow-up}

Although there was no significant difference in LV ejection fraction between the two groups, LVEDV and LVESV were larger in patients with recurrent VT/VF than patients without recurrent VT/VF (Table 1). Receiver operating characteristic curve analysis was used to calculate the area under the curve for the optimal volume of LVEDV and LVESV in predicting the recurrence of VT/VF. Only LVEDV > $163.5 \mathrm{~mL}$ had significant predictive value for the recurrence of VT/VF (area under the curve: $0.602, p=0.041$ ). Moreover, the percentage of patients with LVEDV $>163.5 \mathrm{~mL}$ was significantly higher in patients with recurrent VT/VF than patients without recurrent VT/VF $(p=0.010)$. Receiver operating characteristic curve analysis was also used to calculate the area under the curve for the optimal duration of QRS width in predicting the recurrence of VT/VF. QRS width over $125 \mathrm{msec}$ had the best predictive value, although it did not reach statistical significance (area under the curve: 0.563, $p=0.209)$. Eighty-six patients (59.7\%) had followup echocardiograms at a median follow-up period of 939.5 days. Notably, LVEDV at follow-up were significantly larger in patients with recurrent VT/VF than patients without recurrent VT/VF (191.00 \pm 76.61 vs. $158.15 \pm 57.82 \mathrm{~mL}, p=0.028)$. Moreover, there was a trend, but not statistically significant that patients with no recurrent VT/VF were more likely to have reverse remodeling of LVEDV more prominently $(-21.73 \pm 54.49$ vs. $-0.74 \pm 55.72 \mathrm{~mL}, p=0.083)$ and more frequently (58.3\% vs. $44.7 \%, p=0.266)$ compared with patients with recurrent VT/VF (Fig. 2). The LV ejection fraction at follow-up remained similar between patients with recurrent VT/VF and patients without recurrent VT/VF ( $47.2 \pm 16.3$ vs. $46.7 \pm 16.2 \%, p=0.885)$.

By univariate Cox regression analyses, LV ejection fraction $\leq 30 \%(p=0.031)$, larger LVEDV $(p=0.028)$, LVEDV > $163.5 \mathrm{~mL}(p=0.012)$, and larger LVESV $(p=0.025)$ were significant predictors for VT/VF recurrence during follow-up (Table 2). QRS width $>125 \mathrm{msec}$ was also a significant predictor for VT/VF recurrence during follow-up $(p=0.049)$. There was a trend, but not statistically significant that anti-arrhythmics had a protective role for VT/ VF recurrence (hazard ratio: 0.611, 95\% confidence interval: $0.298 \sim 1.252, p=0.178$ ) (Table 2). However, only LVEDV > $163.5 \mathrm{~mL}$ (hazard ratio: 2.549 , 95\% confidence interval: $1.249 \sim 5.201, p=0.010)$ and QRS width > $125 \mathrm{msec}$ (hazard ratio: 2.173, 95\% confidence interval: $1.030 \sim 4.586, p=0.042$ ) were independent predictors for recurrence of VT/VF during follow-up after multivariate adjustment (Table 2) (Fig. 3).

\section{Cardiovascular death and all-cause mortality during follow-up}

The average follow-up periods in the no VT/VF recurrence group and the recurrent VT/VF group were $1019.5 \pm$ 791.4 days and $1266.7 \pm 1028.5$ days, respectively $(p=$ 0.108 ). The 1-year cardiovascular mortality was significant higher in the recurrent VT/VF group than the no recurrence VT/VF group ( $9.4 \%$ vs. $1.1 \%, p=0.028$ ) (Table 1 ). However, there was no significant difference in the 1-year all-cause mortality between the two groups (9.4\% vs. $5.5 \%$, $p=0.499)$.

\section{Discussion}

This study identifies and reports $36.8 \%$ of ICD recipients for secondary prevention having recurrent $\mathrm{VT} / \mathrm{VF}$ at a mean follow-up of $1110.5 \pm 860.6$ days. LVEDV $>163.5 \mathrm{~mL}$ 
Table 1 Baseline characteristics of study patients

\begin{tabular}{|c|c|c|c|}
\hline & $\begin{array}{l}\text { No VT/NF group } \\
(N=91)\end{array}$ & $\begin{array}{l}\text { Recurrent VTNF group } \\
(N=53)\end{array}$ & $p$ value \\
\hline \multicolumn{4}{|l|}{ General demographics } \\
\hline Age (year) & $63 \pm 14$ & $60 \pm 13$ & 0.166 \\
\hline Male gender & $73(80.2)$ & $41(77.4)$ & 0.419 \\
\hline BMI & $25.5 \pm 4.7$ & $25.1 \pm 3.8$ & 0.686 \\
\hline \multicolumn{4}{|l|}{ Heart disease } \\
\hline Congenital heart & $4(4.4)$ & $0(0)$ & 0.297 \\
\hline CAD & $51(56.0)$ & $29(54.7)$ & 0.979 \\
\hline Treatment in CAD patients & & & 0.020 \\
\hline CABG & $5(9.8)$ & $10(34.5)$ & \\
\hline $\mathrm{PCl}$ & $41(80.4)$ & $18(62.1)$ & \\
\hline Medical treatment & $5(9.8)$ & $1(3.4)$ & \\
\hline DCM & $16(17.6)$ & $11(20.8)$ & 0.397 \\
\hline $\mathrm{HOCM}$ & $4(4.4)$ & $2(1.9)$ & 0.391 \\
\hline Idiopathic VF & $8(8.8)$ & $3(5.7)$ & 0.370 \\
\hline Brugada syndrome & $3(3.3)$ & $3(5.7)$ & 0.388 \\
\hline Long QT syndrome & $4(4.4)$ & $3(3.8)$ & 0.391 \\
\hline Valvular heart disease & $8(8.8)$ & $8(15.1)$ & 0.187 \\
\hline \multicolumn{4}{|l|}{ Comorbidity } \\
\hline Hypertension & $52(57.1)$ & $26(49.1)$ & 0.222 \\
\hline Diabetes & $24(26.4)$ & $20(37.7)$ & 0.108 \\
\hline Prior stroke & $10(11.0)$ & $7(13.2)$ & 0.441 \\
\hline Hyperlipidemia & $28(30.8)$ & $14(26.4)$ & 0.360 \\
\hline ESRD & $6(6.6)$ & $5(9.4)$ & 0.377 \\
\hline Atrial fibrillation (\%) & & & 0.634 \\
\hline No & 67 (73.6) & $40(75.5)$ & \\
\hline Paroxysmal & $17(18.7)$ & $11(20.8)$ & \\
\hline Persistent & $7(7.7)$ & $2(3.8)$ & \\
\hline Heart failure & & & 0.865 \\
\hline Without heart failure & $29(31.9)$ & $16(30.2)$ & \\
\hline NYHA functional class I & $16(17.6)$ & $11(20.8)$ & \\
\hline NYHA functional class II & $27(29.7)$ & $13(24.5)$ & \\
\hline NYHA functional class III & $14(15.4)$ & $8(15.1)$ & \\
\hline NYHA functional class IV & $5(5.5)$ & $5(9.4)$ & \\
\hline Diastolic function (\%) & & & 0.322 \\
\hline Normal & $11(12.9)$ & $11(22.9)$ & \\
\hline Stage I & $40(47.1)$ & $24(50.0)$ & \\
\hline Stage II & $14(31.8)$ & $8(22.9)$ & \\
\hline Stage III & $5(8.2)$ & $5(4.2)$ & \\
\hline QRS length (msec) & $115.3 \pm 30.7$ & $121.8 \pm 32.3$ & 0.231 \\
\hline$\angle B B B$ & $2(2.2)$ & $4(7.5)$ & 0.121 \\
\hline \multicolumn{4}{|l|}{ Medications } \\
\hline ACEI/ARB & $63(69.2)$ & $32(60.4)$ & 0.184 \\
\hline ß-blocker & $59(64.8)$ & $28(52.8)$ & 0.107 \\
\hline
\end{tabular}


Table 1 Baseline characteristics of study patients (Continued)

\begin{tabular}{|c|c|c|c|}
\hline Bisoprolol (the average dose) (mg) & $3.5 \pm 2.2$ & $3.2 \pm 1.5$ & 0.530 \\
\hline Carvedilol (the average dose) (mg) & $17.2 \pm 10.0$ & $9.1 \pm 13.1$ & 0.651 \\
\hline Diuretic & $30(33.0)$ & $15(28.3)$ & 0.348 \\
\hline Statin & $29(31.9)$ & $20(37.7)$ & 0.473 \\
\hline Spironolactone & $11(12.1)$ & $11(20.8)$ & 0.125 \\
\hline Anti-platelet agent & $54(59.3)$ & $29(54.7)$ & 0.604 \\
\hline Warfarin & $12(13.2)$ & $9(17.0)$ & 0.626 \\
\hline NOAC & $3(3.3)$ & $0(0)$ & 0.297 \\
\hline \multicolumn{4}{|l|}{ Anti-arrhythmic medications } \\
\hline Amiodarone & $62(68.1)$ & $33(62.3)$ & 0.295 \\
\hline The average dose (mg) & $196.8 \pm 85.4$ & $214.3 \pm 103.3$ & 0.371 \\
\hline Dronedarone & $2(2.2)$ & $0(0)$ & 0.532 \\
\hline The average dose (mg) & 800 & & \\
\hline Quinidine & $2(2.2)$ & $0(0)$ & 0.532 \\
\hline The average dose (mg) & 600 & & \\
\hline Mexiletine & $2(2.2)$ & $0(0)$ & 0.532 \\
\hline The average dose (mg) & 300 & & \\
\hline Sotalol & $1(1.1)$ & $0(0)$ & 1.000 \\
\hline The average dose (mg) & 160 & & \\
\hline CRT-D & $1(1.1)$ & $3(5.7)$ & 0.108 \\
\hline ICD chamber & & & 0.731 \\
\hline Single & $51(56.0)$ & $28(52.8)$ & \\
\hline Dual & $40(44.0)$ & $25(47.2)$ & \\
\hline Ventricular-dependent pacing & $4(4.4)$ & $1(1.9)$ & 0.428 \\
\hline Hemodynamic condition & & & 0.590 \\
\hline Stable & $60(65.9)$ & $32(60.4)$ & \\
\hline Unstable & $31(34.1)$ & $21(39.6)$ & \\
\hline Lowest VT-detection zone (bpm) & $165.9 \pm 13.2$ & $165.4 \pm 10.5$ & 0.865 \\
\hline Lowest VF-detection zone (bpm) & $209.2 \pm 13.5$ & $208.2 \pm 15.8$ & 0.773 \\
\hline Post VT ablation (\%) & $6(6.6)$ & $9(17.0)$ & 0.087 \\
\hline Success & $3(50)$ & $3(33.3)$ & 0.518 \\
\hline Failure & $3(50)$ & $6(66.7)$ & \\
\hline Combination of VT ablation plus medications (anti-arrhythmics and B-blocker) & $6(6.6)$ & $6(11.3)$ & 0.358 \\
\hline \multicolumn{4}{|l|}{ LV systolic function } \\
\hline LVEF (\%) & $49.5 \pm 16.7$ & $44.6 \pm 18.5$ & 0.105 \\
\hline LVEDV (mL) & $162.0 \pm 68.1$ & $186.4 \pm 84.5$ & 0.060 \\
\hline LVEDV > $163.5 \mathrm{~mL}$ & $36(40.0)$ & $33(62.3)$ & 0.010 \\
\hline LVESV (mL) & $88.2 \pm 61.3$ & $111.4 \pm 81.6$ & 0.055 \\
\hline 1-year CV mortality & $1(1.1)$ & $5(9.4)$ & 0.028 \\
\hline 1-year all-cause mortality & $5(5.5)$ & $5(9.4)$ & 0.499 \\
\hline Follow-up time (days) & $1019.5 \pm 791.4$ & $1266.7 \pm 1028.5$ & 0.108 \\
\hline
\end{tabular}

\footnotetext{
Data are expressed as mean \pm SD or as number (percentage)
}

${ }^{*} A b b r e v i a t i o n s: V T$ ventricular tachycardia, VF ventricular fibrillation, $B M I$ body mass index, CAD coronary artery disease, CABG coronary artery bypass graft surgery, $P C l$ percutaneous coronary intervention, DCM dilated cardiomyopathy, HOCM hypertrophic obstructive cardiomyopathy, RVOT right ventricular outflow tract, ESRD end stage renal disease, $L V E F$ left ventricular ejection fraction, $L V E D V$ left ventricular end diastolic volume, LVESV left ventricular end systolic volume, NYHA New York Heart Association, LBBB left bundle branch block, NOAC non-vitamin K oral anticoagulants, CRT-D, cardiac resynchronization therapy-defibrillator, ACEI angiotensin-converting-enzyme inhibitor, $A R B$ angiotensin receptor blocker, $C V$ cardiovascular 


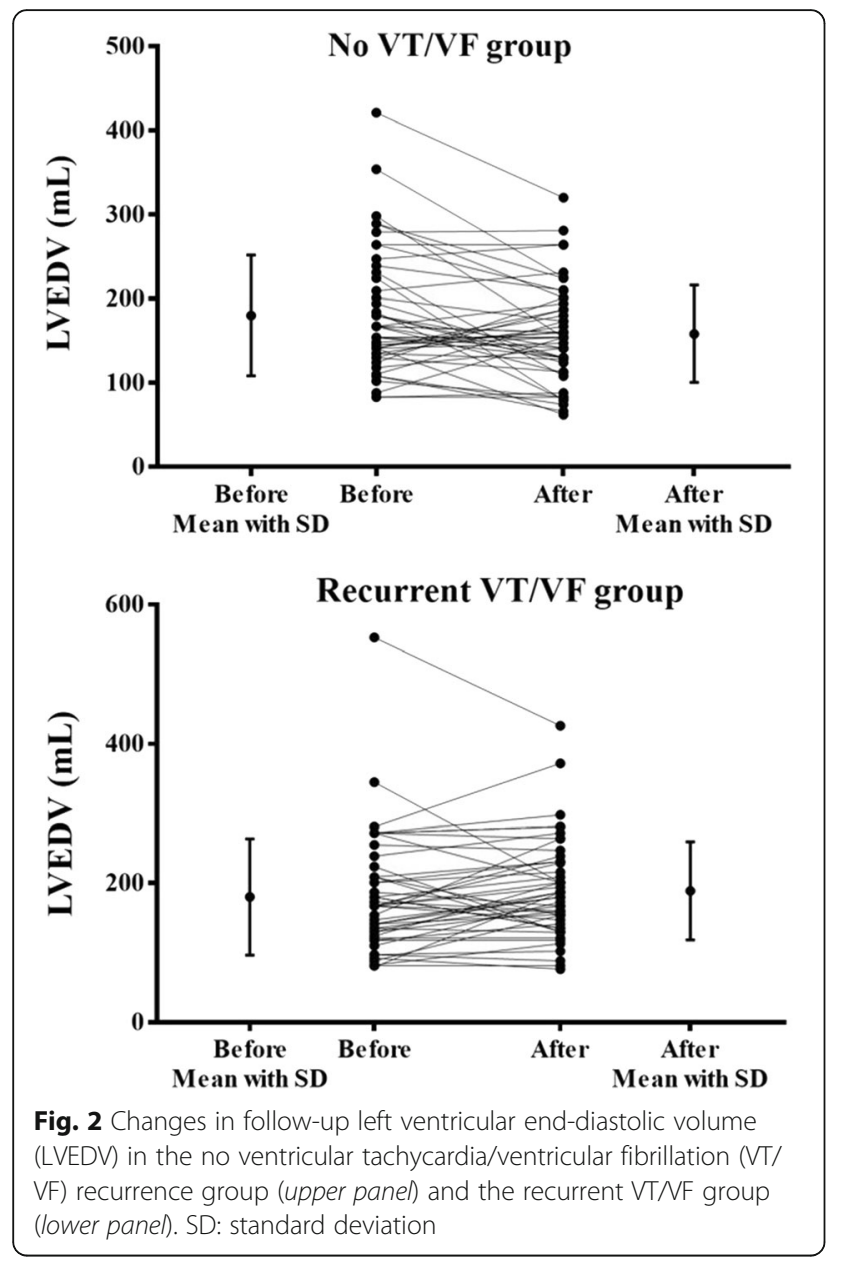

and QRS width $>125$ msec were independent predictors for recurrence of VT/VF during follow-up after multivariable adjustment.

In the multicentre COMFORT trial, Zaman et al. reported that secondary prevention ICD recipients had more recurrent VT/VF than primary prevention ICD recipients (37.1\% vs. $19.9 \%$ at 2-year follow-up) [13]. In our study, $36.8 \%$ of ICD recipients for secondary prevention had recurrent VT/VF, which was comparable to the recurrent rate reported in the COMFORT trial.

Ventricular tachyarrhythmia is an important cause of cardiovascular mortality, morbidity and heart failure hospitalization in a wide variety of heart diseases [14]. Implantable cardioverter defibrillator is now widely used in patients who survive sustained VT or VF, or who are at high risk for sudden cardiac death $[1,15]$. Although ICDs can decrease risk of arrhythmic death, long-term total mortality still depends on the severity of the underlying heart diseases and associated comorbidities with the increase in nonarrhythmic deaths completely offsetting the decrease in arrhythmic deaths [14]. In our study, cardiovascular mortality was significant higher in the recurrent VT/VF group than the no recurrence VT/VF group, whereas there was no difference in the all-cause mortality (including nonarrhythmic deaths) between the two groups.

According to current guideline, LV ejection fraction has been selected as the most important criteria for ICD implantation in primary and secondary prevention settings [1]. However, several studies have shown improvement of LV ejection fraction during follow-up in primary prevention ICD recipients $[16,17]$. Notably, Kini et al. reported that approximately $25 \%$ of primary prevention ICD recipients may no longer meet guideline indications for ICD use at

Table 2 Univariate and multivariate Cox regression analyses in terms of VTNF recurrence

\begin{tabular}{|c|c|c|c|c|c|c|}
\hline \multirow[b]{2}{*}{ Variables } & \multicolumn{3}{|c|}{ Univariate analysis } & \multicolumn{3}{|c|}{ Multivariate analysis } \\
\hline & Hazard ratio & $95 \% \mathrm{Cl}$ & $p$-value & Hazard ratio & $95 \% \mathrm{Cl}$ & $p$-value \\
\hline Atrial fibrillation (paroxysmal and persistent) & 1.095 & $0.584 \sim 2.052$ & 0.777 & & & \\
\hline LVEF (\%) & 0.986 & $0.970 \sim 1.001$ & 0.074 & & & \\
\hline LVEF $\leqq 30 \%$ & 1.995 & $1.064 \sim 3.740$ & 0.031 & & & \\
\hline LVEDV (mL) & 1.004 & $1.000 \sim 1.007$ & 0.028 & & & \\
\hline LVEDV > $163.5 \mathrm{~mL}$ & 2.042 & $1.170 \sim 3.562$ & 0.012 & 2.549 & $1.249 \sim 5.201$ & 0.010 \\
\hline LVESV (mL) & 1.004 & $1.001 \sim 1.008$ & 0.025 & & & \\
\hline Heart failure NYHA functional class $\geqq 3$ & 1.293 & $0.688 \sim 2.430$ & 0.425 & & & \\
\hline QRS width (msec) & 1.008 & $1.000 \sim 1.016$ & 0.059 & & & \\
\hline QRS width > $125 \mathrm{msec}$ & 2.067 & $1.003 \sim 4.260$ & 0.049 & 2.173 & $1.030 \sim 4.586$ & 0.042 \\
\hline Ischemic cardiomyopathy & 0.998 & $0.580 \sim 1.717$ & 0.995 & & & \\
\hline Dilated cardiomyopathy & 0.851 & $0.437 \sim 1.655$ & 0.634 & & & \\
\hline Anti-arrhythmic drugs & 0.611 & $0.298 \sim 1.252$ & 0.178 & & & \\
\hline Post VT ablation & 0.844 & $0.272 \sim 2.615$ & 0.768 & & & \\
\hline
\end{tabular}

${ }^{*}$ Abbreviations: $V T$ ventricular tachycardia, $V F$ ventricular fibrillation, $C l$ confidence interval, $L V E F$ left ventricular ejection fraction, $L V E D V$ left ventricular end diastolic volume, LVESV left ventricular end systolic volume, NYHA New York Heart Association 


\section{VT/VF occurrence}

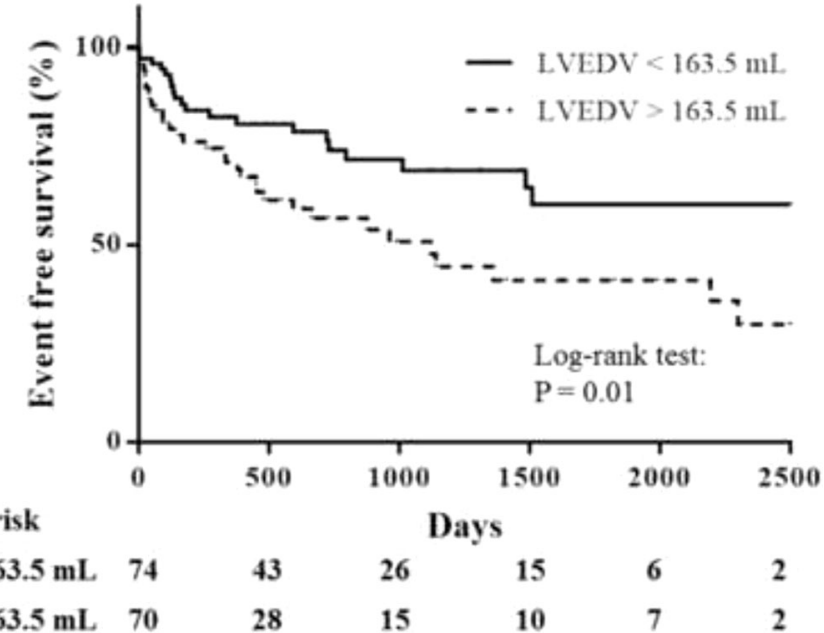

Fig. 3 Kaplan-Meier plot proportion of patients free of recurrent ventricular tachycardia/ventricular fibrillation (VTNF) events after implant stratified by left ventricular end-diastolic volume (LVEDV) of $163.5 \mathrm{~mL}$

the time of generator replacement due to improvement of LV ejection fraction, and these ICD recipients received subsequent ICD therapies at a significantly lower rate [16]. Additionally, in MADIT-CRT trial, patients who achieve LV ejection fraction normalization ( $>50 \%$ ) during follow-up have very low absolute and relative risk of ventricular tachyarrhythmias and a favorable clinical course within 2.2 years of follow-up, and downgrade from cardiac resynchronization therapy-defibrillator to cardiac resynchronization therapy-pacemaker at the time of battery depletion for cost savings could be considered in these patients if no ventricular tachyarrhythmias have recurred [17]. However, ICD therapy is reimbursed only for secondary prevention in many countries, and limited data regarding LV remodeling as predictor for recurrent VT/VF are available for secondary prevention ICD recipients. Klein et al. reported that LV ejection fraction $<40 \%$, permanent atrial fibrillation and QRS duration $>150 \mathrm{msec}$ are independent predictors for VT/VF recurrence in ICD recipients predominantly for secondary prevention [8]. However, Freedberg et al. reported that LV ejection fraction was not a predictor for subsequent ICD therapy in secondary prevention ICD recipients [9]. In our study for secondary prevention ICD recipients, LVEDV > $163.5 \mathrm{~mL}$ and QRS width $>125 \mathrm{msec}$ were independent predictors for recurrence of VT/VF during followup after multivariable adjustment (including LV ejection fraction, atrial fibrillation and heart failure status), and LVEF $\leq 30 \%$ was no longer a predictor after multivariable adjustment (Table 2).

\section{Study limitations}

There are several limitations in this study. Firstly, this retrospective analysis bears the inherent limitations of these types of studies. Secondly, LVEDVs were estimated by M-mode echocardiography and were corrected by two-dimensional guided biplanar Simpson's method of discs for measurement and this quantified method has been proven to be an acceptable method to evaluate LV volume. Moreover, there was a trend that patients with no recurrent VT/VF were more likely to have reverse remodeling of LVEDV during follow-up compared with patients with recurrent VT/VF (Fig. 2).

\section{Conclusions}

LV remodeling and QRS width $>125$ msec were independent predictors for recurrence of VT/VF during follow-up in secondary prevention ICD recipients under optimal medical therapy, independent of LV ejection fraction.

\section{Abbreviations}

ICD: Implantable cardioverter defibrillator; LV: Left ventricular; LVEDV: LV enddiastolic volume; LVESV: LV end-systolic volume; VTNF: Ventricular tachycardia/ Ventricular fibrillation

\section{Acknowledgements \\ Not applicable. \\ Funding \\ None.}

Availability of data and materials

The datasets used and/or analysed during the current study available from the corresponding author on reasonable request.

\section{Author's contributions}

WCL, HCC, YLC, THT, KLP, YSL and MCC designed the research; WCL, HCC and MCC performed the research; WCL, HCC, YLC and MCC analyzed the data; WCL and MCC wrote the paper. All authors read and approved the final manuscript.

Competing interests

The authors declare that they have no competing interests. 


\section{Consent for publication}

Not applicable.

\section{Ethics approval and consent to participate}

The study protocol conforms to the ethical guidelines of the 1975 Declaration of Helsinki and was approved by the Institutional Review Board of Chang Gung Memorial Hospital (104-8143B) and the need for written informed consent from all participants was granted an exemption from Institutional Review Board of Chang Gung Memorial Hospital as this study included only retrospective noninvasive data analysis.

\section{Author details}

'Division of Cardiology, Department of Internal Medicine, Kaohsiung Chang Gung Memorial Hospital, Chang Gung University College of Medicine, 123 Ta Pei Road, Niao Sung District, Kaohsiung City 83301, Taiwan. ${ }^{2}$ Division of Cardiology, Chang Gung Memorial Hospital, Chiayi, Taiwan.

Received: 9 June 2016 Accepted: 15 November 2016 Published online: 21 November 2016

\section{References}

1. Russo AM, Stainback RF, Bailey SR, Epstein AE, Heidenreich PA, Jessup M, Kapa S, Kremers MS, Lindsay BD, Stevenson LW. ACCF/HRS/AHA/ASE/HFSA/ SCAI/SCCT/SCMR 2013 appropriate use criteria for implantable cardioverterdefibrillators and cardiac resynchronization therapy: a report of the American College of Cardiology Foundation appropriate use criteria task force, Heart Rhythm Society, American Heart Association, American Society of Echocardiography, Heart Failure Society of America, Society for Cardiovascular Angiography and Interventions, Society of Cardiovascular Computed Tomography, and Society for Cardiovascular Magnetic Resonance. J Am Coll Cardiol. 2013;61:1318-68.

2. Bardy $\mathrm{GH}$, Lee $\mathrm{KL}$, Mark DB, Poole JE, Packer DL, Boineau R, Domanski M, Troutman C, Anderson J, Johnson G, McNulty SE, Clapp-Channing N, Davidson-Ray LD, Fraulo ES, Fishbein DP, Luceri RM, Ip JH. Sudden Cardiac Death in Heart Failure Trial (SCD-HeFT) investigators. Amiodarone or an implantable cardioverter-defibrillator for congestive heart failure. N Engl J Med. 2005;352:225-37.

3. Buxton AE, Lee KL, Fisher JD, Josephson ME, Prystowsky EN, Hafley G. A randomized study of the prevention of sudden death in patients with coronary artery disease. Multicenter Unsustained Tachycardia Trial Investigators. N Engl J Med. 1999;341:1882-90.

4. Kadish A, Dyer A, Daubert JP, Quigg R, Estes NA, Anderson KP, Calkins H, Hoch D, Goldberger J, Shalaby A, Sanders WE, Schaechter A, Levine JH. Defibrillators in Non-Ischemic Cardiomyopathy Treatment Evaluation (DEFINITE) Investigators. Prophylactic defibrillator implantation in patients with nonischemic dilated cardiomyopathy. N Engl J Med. 2004;350:2151-8.

5. Moss AJ, Zareba W, Hall WJ, Klein H, Wilber DJ, Cannom DS, Daubert JP, Higgins SL, Brown MW, Andrews ML. Multicenter Automatic Defibrillator Implantation Trial II Investigators. Prophylactic implantation of a defibrillator in patients with myocardial infarction and reduced ejection fraction. $N$ Engl J Med. 2002;346:877-83.

6. Bristow MR, Saxon LA, Boehmer J, Krueger S, Kass DA, De Marco T, Carson P, DiCarlo L, DeMets D, White BG, DeVries DW, Feldman AM. Comparison of Medical Therapy, Pacing, and Defibrillation in Heart Failure (COMPANION) Investigators. Cardiac-resynchronization therapy with or without an implantable defibrillator in advanced chronic heart failure. N Engl J Med. 2004;350:2140-50.

7. Moss AJ, Hall WJ, Cannom DS, Daubert JP, Higgins SL, Klein H, Levine JH, Saksena S, Waldo AL, Wilber D, Brown MW, Heo M. Improved survival with an implanted defibrillator in patients with coronary disease at high risk for ventricular arrhythmia. Multicenter Automatic Defibrillator Implantation Trial Investigators. N Engl J Med. 1996;335:1933-40.

8. Klein G, Lissel C, Fuchs AC, Gardiwal A, Oswald H, Desousa M, Pichlmaier AM, Lichtinghagen R, Geerlings H, Lippolt P, Niehaus M, Drexler H, Korte T. Predictors of VTNF-occurrence in ICD patients: results from the PROFITStudy. Europace. 2006:8:618-24.

9. Freedberg NA, Hill JN, Fogel RI, Prystowsky EN, CARE Group. Recurrence of symptomatic ventricular arrhythmias in patients with implantable cardioverter defibrillator after the first device therapy: implications for antiarrhythmic therapy and driving restrictions. J Am Coll Cardiol. 2001;37:1910-5.
10. Frigerio M, Roubina E. Drugs for left ventricular remodeling in heart failure Am J Cardiol. 2005;96:10L-8L.

11. Crawford MH, Grant D, O'Rourke RA, Starling MR, Groves BM. Accuracy and reproducibility of new M-mode echocardiographic recommendations for measuring left ventricular dimensions. Circulation. 1980;61:137-43.

12. Lang RM, Bierig M, Devereux RB, Flachskampf FA, Foster E, Pellikka PA, Picard MH, Roman MJ, Seward J, Shanewise JS, Solomon SD, Spencer KT, Sutton MS, Stewart WJ. Recommendations for chamber quantification: A report from the American Society of Echocardiography's Guidelines and Standards Committee and the Chamber Quantification Writing Group, developed in conjunction with the European Association of Echocardiography, a branch of the European Society of Cardiology. J Am Soc Echocardiogr. 2005;18:1440-63.

13. Zaman S, Sivagangabalan G, Chik W, Stafford W, Hayes J, Denman R, Young $\mathrm{G}$, Sanders $\mathrm{P}$, Kovoor $\mathrm{P}$. Ventricular tachyarrhythmia recurrence in primary versus secondary implantable cardioverter-defibrillator patients and role of electrophysiology study. J Interv Card Electrophysiol. 2014;41:195-202.

14. Dorian P, Hohnloser SH, Thorpe KE, Roberts RS, Kuck KH, Gent M, Connolly SJ. Mechanisms underlying the lack of effect of implantable cardioverterdefibrillator therapy on mortality in high-risk patients with recent myocardial infarction: insights from the Defibrillation in Acute Myocardial Infarction Trial (DINAMIT). Circulation. 2010;122:2645-52.

15. Goldenberg I, Gillespie J, Moss AJ, Hall WJ, Klein H, McNitt S, Brown MW, Cygankiewicz I, Zareba W. Long-term benefit of primary prevention with an implantable cardioverter-defibrillator: an extended 8-year follow-up study of the Multicenter Automatic Defibrillator Implantation Trial II. Circulation. 2010;122:1265-71.

16. Kini V, Soufi MK, Deo R, Epstein AE, Bala R, Riley M, Groeneveld PW, Shalaby A, Dixit S. Appropriateness of primary prevention implantable cardioverterdefibrillators at the time of generator replacement: are indications still met? J Am Coll Cardiol. 2014;63:2388-94.

17. Ruwald MH, Solomon SD, Foster E, Kutyifa V, Ruwald AC, Sherazi S, McNitt S, Jons C, Moss AJ, Zareba W. Left ventricular ejection fraction normalization in cardiac resynchronization therapy and risk of ventricular arrhythmias and clinical outcomes: results from the Multicenter Automatic Defibrillator Implantation Trial With Cardiac Resynchronization Therapy (MADIT-CRT) trial. Circulation. 2014;130:2278-86.

\section{Submit your next manuscript to BioMed Central and we will help you at every step:}

- We accept pre-submission inquiries

- Our selector tool helps you to find the most relevant journal

- We provide round the clock customer support

- Convenient online submission

- Thorough peer review

- Inclusion in PubMed and all major indexing services

- Maximum visibility for your research

Submit your manuscript at www.biomedcentral.com/submit
) Biomed Central 